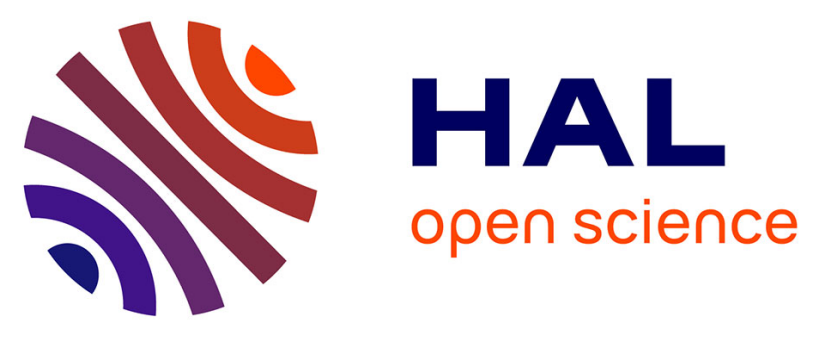

\title{
High-speed video analysis improves the accuracy of spinal cord compression measurement in a mouse contusion model
}

Marion Fournely, Yvan Petit, Eric Wagnac, Jérôme Laurin, Virginie Callot, Pierre-Jean Arnoux

\section{To cite this version:}

Marion Fournely, Yvan Petit, Eric Wagnac, Jérôme Laurin, Virginie Callot, et al.. High-speed video analysis improves the accuracy of spinal cord compression measurement in a mouse contusion model. Journal of Neuroscience Methods, 2018, 293, pp.1-5. 10.1016/j.jneumeth.2017.09.007 · hal-01703435

\section{HAL Id: hal-01703435 \\ https://hal-amu.archives-ouvertes.fr/hal-01703435}

Submitted on 10 Apr 2018

HAL is a multi-disciplinary open access archive for the deposit and dissemination of scientific research documents, whether they are published or not. The documents may come from teaching and research institutions in France or abroad, or from public or private research centers.
L'archive ouverte pluridisciplinaire HAL, est destinée au dépôt et à la diffusion de documents scientifiques de niveau recherche, publiés ou non, émanant des établissements d'enseignement et de recherche français ou étrangers, des laboratoires publics ou privés. 
High-speed video analysis improves the accuracy of spinal cord compression measurement in a mouse contusion model

3 Jérôme Laurin - ISM - Institut des Sciences du Mouvement Etienne Jules Marey

4 Marion Fournely - LBA UMR T24 - Laboratoire de Biomécanique Appliquée

5 Yvan Petit - Hopital du Sacré-Coeur de Montréal, ETS - Ecole de Technologie Supérieure

$6 \quad$ [Montréal]

$7 \quad$ Eric Wagnac - LBA UMR T24 - Laboratoire de Biomécanique Appliquée

8 Virginie Callot - CRMBM - Centre de résonance magnétique biologique et médicale

9 Pierre-Jean Arnoux - LBA UMR T24 - Laboratoire de Biomécanique Appliquée 


\section{Abstract}

\section{Background:}

3 Animal models of spinal cord injuries aim to utilize controlled and reproducible conditions. However,

4 a literature review reveals that mouse contusion studies using equivalent protocols may show large disparities in the observed impact force vs. cord compression relationship. The overall purpose of this

6 study was to investigate possible sources of bias in these measurements. The specific objective was to

7 improve spinal cord compression measurements using a video-based setup to detect the impactor-

8 spinal cord time-to-contact.

New Method:

10 A force-controlled $30 \mathrm{kDyn}$ unilateral contusion at $\mathrm{C} 4$ vertebral level was performed in six mice with 11 the Infinite Horizon impactor $(\mathrm{IH})$. High-speed video was used to determine the time-to-contact between the impactor tip and the spinal cord and to compute the related displacement of the tip into the tissue: the spinal cord compression and the compression ratio.

\section{Results \& Comparison with Existing Method(s)}

Delayed time-to-contact detection with the IH device led to an underestimation of the cord compression. Compression values indicated by the $\mathrm{IH}$ were $64 \%$ lower than those based on video analysis $(0.33 \mathrm{~mm}$ vs. $0.88 \mathrm{~mm})$. Consequently, the mean compression ratio derived from the device was underestimated when compared to the value derived from video analysis (22\% vs. $61 \%$ ).

\section{Conclusions}

Default time-to-contact detection from the IH led to significant errors in spinal cord compression assessment. Accordingly, this may explain some of the reported data discrepancies in the literature. The proposed setup could be implemented by users of contusion devices to improve the quantative description of the primary injury inflicted to the spinal cord.

Keywords: Spinal cord injury, Mouse model, Contusion device, Compression ratio, High-speed video analysis. 


\section{1}

2

\section{Introduction}

Experimental animal Spinal Cord Injury (SCI) models aim to reproduce clinical features of human SCI and to control all parameters of the trauma in order to obtain consistent and reproducible injuries. Most human SCIs are caused by blunt trauma induced by disk or bone displacement into the medullar canal (Sekhon and Fehlings, 2001). In animal studies, the most common way to reproduce this type of injury is the contusion model (Sharif-Alhoseini et al., 2017) which consists of a dynamic compression of the spinal cord.

Three commercially-available devices are proposed for performing spinal cord contusion in rodent models. The NYU-MACSIS Impactor (Gruner, 1992) drops a weight from a pre-defined distance onto the exposed spinal cord while the ESCID (Ohio State University) (Jakeman et al., 2009) and the Infinite Horizon (IH, Precision Systems and Instrumentation) (Scheff et al., 2003) impactors drive a cylindrical tip onto the exposed spinal cord until a user-define displacement (ESCID) or force value $(\mathrm{IH})$ is reached. The ESCID and the IH then estimate the undefined force or displacement imposed on the spinal cord, respectively. The ESCID and IH devices were independently reported to produce reliable and reproducible lesions (Sharif-Alhoseini et al., 2017). However, replication studies of rodent SCI models using both devices alternately have shown great variability in both the injury morphologies and the corresponding neurological effects (Steward et al., 2012). Moreover, similar protocols have led to large differences between force/displacement outcomes. For example, Myers et al. (2014) performed contusions at T9/T10 on C57Bl/6 female adult mice using the IH device with a force input of $50 \mathrm{kDyn}$ and obtained a mean displacement of $0.59 \mathrm{~mm}$ whereas Ma et al. (2001) performed contusions on the same animal model with the ESCID device and only required a displacement input of $0.3 \mathrm{~mm}$ in order to obtain a mean force of 50kDyn. Furthermore, EngesserCesar et al. (2005) reported significant differences in displacement outcomes for the same force set point value when using two identical IH devices. Potential experimental factors explaining these differences are not identified in the related paper but the lack of sensitivity of the force sensor at the point-of-contact is probably the most important source of variability in this context. 
1 Several factors could account for this lack of consistency in force/displacement outcomes. Failure of

2 the vertebral stabilization system has been frequently mentioned in this regard (Sharif-Alhoseini et al., 3 2017). The ESCID and IH devices also use different force thresholds to detect contact between 4 impactor tip and the cord (Jakeman et al., 2009; Scheff et al., 2003). In addition, the impactor tip 5 geometry or positioning might also introduce biases. According to Basso et al. (2006), a variation of $60.1 \mathrm{~mm}$ in impactor displacement or $10 \mathrm{kDyn}$ in the applied force may lead to important differences in 7 behavioral outcomes. Therefore, there is a crucial need to identify and report these sources of bias and 8 define sustainable mechanical criteria to achieve reproducible injuries.

9 The main objective of this work was to propose an video-based system to detect impactor-spinal cord 10 time-to-contact and thus, to improve the accuracy of the spinal cord compression measurement. This 11 system was designed to also allow qualitative assessment of the vertebral clamping system 12 effectiveness. 


\section{1}

\section{Materials and methods}

Experiments were performed on a total of six C57BL/6 mice using a protocol approved by our institutional ethics board "Comité d'Ethique Marseille ( $\left.{ }^{\circ} 14\right)$ " (Approval number 18-26072012).

\section{Morphological measurements}

Anatomical magnetic resonance acquisitions of Callot et al. (2007) on female 17-24 weeks old C57BL/6 mice $(n=9)$ were retrospectively used to evaluate the mean lateral anteroposterior diameter of the spinal cord $\left(D \emptyset_{A P}\right.$ in mm). Transverse images at the $\mathrm{C} 4$ vertebral level were acquired at $11.75 \mathrm{~T}$ (Brucker, Ettlingen, Germany) with in-plane spatial resolution of 0.1 x $0.1 \mathrm{~mm}$. Measurements were performed manually along the dorsoventral axis of the cord, on the left side and where the diameter is maximal (Figure 1A). Measurements were made using ImageJ (Schneider et al., 2012) and repeated three times to evaluate intra-operator repeatability. Maximal right-left diameter was also evaluated.

\section{Experimental model}

The spinal cord contusion was performed using the Infinite Horizon impactor device (IH-0400, version 5.0, Precision Systems \& Instrumentation, Lexington, KY, USA) equipped with a IH custommade $0.6 \mathrm{~mm}$ diameter impacting tip. Before the spinal cord impact, mice (Female, 17-30 weeks old, 20.1-23.5g) were anesthetized and a midline longitudinal skin incision was performed over the upper cervical area. Vertebral muscles were dissected to reach the posterior vertebral elements from C3 to C5 vertebrae. A partial dorsolateral laminectomy of the left C4 lamina (vertebral arch) was then performed to expose the dura mater, without removing or affecting it. More precisely, only half of the left C4 lamina was removed without affecting the $\mathrm{C} 4$ spinous process. In this way, the exposed area of the spinal cord was large enough to avoid touching the surrounding vertebral elements with the impacting tip (Figure 1B-1C). The spine was immobilized with two clamps (provided with the impactor positioned on C3 and C5 vertebrae. The impacting tip was placed above the exposed spinal cord. The tip placement was the most lateral as possible to only affect the lateral part of the spinal cord without touching the transverse processes during the contusion sequence, which could change the impact force. The impacting force was set at $30 \mathrm{kDyn}(0.3 \mathrm{~N})$ with an impact speed of $0.12 \mathrm{~mm} / \mathrm{ms}$ and no dwell time. This moderate impacting force was used to proscribe mortality induced by strong 
1 respiration dysfunction (due to damage to the $\mathrm{C} 4$ ventral horn neurons innervating the diaphragm via

2 the phrenic nerve). Muscles and skin were then sutured. No hemorrhage was observed during or after

3 the surgery. The IH device monitored the displacement of the tip $\left(\operatorname{Disp}_{I H}(t)\right.$ in $\left.\mathrm{mm}\right)$ and the force

4 measured by the load cell at a frequency of $3 \mathrm{kHz}$ (Figure 1D). At the end of the contusion process, the device software computed the estimated spinal cord compression $\left(C_{I H}\right.$ in $\left.\mathrm{mm}\right)$, which was defined as the tip displacement from the device-based point-of-contact (20 kDyn contact force threshold) to the point of peak force $\left(F_{I H}\right.$ in $\left.\mathrm{kDyn}\right)$. The corresponding cord compression ratio (\%) was determined by $C R_{I H}=100 \times C_{I H} / D \emptyset_{A P}$.

\section{Video acquisition}

The contusion sequences were filmed with a high-speed camera equipped with a macro lens (AF-S Micro Nikkor 60mm F/2.8G ED, Nikon, Japan). The images were taken from the lateral position, so that the exposed dura, the tip of the impactor, the dorsal parts of the $\mathrm{C} 3$ and $\mathrm{C} 5$ vertebrae and the two clamps were in the field of view (Figure 1C). Two different high-speed cameras were used during the study: the X-VIT LShigh-speed camera (AOS Technologies, Switzerland) at 1000 frames per second (fps) mode for three mice, and the FastCam SA3 (Photron USA, Inc.) at $3000 \mathrm{fps}$ for three other mice. By using the latter equipment, brighter images were obtained at a higher frequency were obtained without modifying the experimental design. The impact of this equipment change on the results accuracy has been assessed (see Results section).

\section{Control of vertebral clamping}

The effectiveness of the IH clamping system was qualitatively assessed from the video recording, using four anatomical and apparatus features. The dorsal parts of the C3 and C5 vertebrae and the two clamps were tracked frame by frame through a template matching analysis based on normalized 2-D cross-correlation using a custom Matlab script (Mathworks, USA).

\section{Video-based contact detection}

The video recording was screened frame by frame by an observer to identify the first occurrence of 
1 be used as a contact indicator. It was then considered that the contact occurred between the selected

2 frame $\left(\right.$ Frame $\left._{n}\right)$ and the previous frame $\left(\right.$ Frame $\left._{n-1}\right)$.

3 Following the same method, the observer was also asked to indicate the first image frame in which the

4 tip began to retract. It was then considered that the tip began to retract between the selected frame

$5 \quad\left(\right.$ Frame $\left._{m}\right)$ and the previous frame $\left(\right.$ Frame $\left._{m-1}\right)$.

6 The time (ms) between initial contact and the tip retraction was determined by $T_{\text {video }}=$

$7 m-n / f_{\text {video }}$, where $f_{\text {video }}$ was the video acquisition frequency in images per millisecond.

8 Resolution uncertainty on $T_{\text {video }}$ was assessed as standard Type B uncertainty with rectangular

9 distribution (JCGM 100:2008, 2008) $: \Delta T_{\text {video }}=1 / f_{\text {video }} \times 1 / \sqrt{3}$

In order to assess the reproducibility of the video-based contact detection method, two additional observers performed the analysis once for the 6 specimens. Observer \#1 also repeated the image frame analysis 3 times on the 6 specimens with a period of 2 months between the analyses.

\section{Corrected compression value and Compression ratio}

The IH impactor displacement data $\left(\operatorname{Disp}_{I H}(t)\right)$ was processed to set the zero displacement and time at the point where the tip retracted. In this coordinate system, the video-based contact occurred at

$16-T_{\text {video }}$ and the corresponding maximal spinal cord compression value is $\operatorname{Disp}_{I H}\left(-T_{\text {video }}\right.$ ) (see Figure 1D). The video-based spinal cord compression value $\left(C_{\text {video }}=\operatorname{Disp}_{I H}\left(-T_{\text {video }}\right)\right)$ was computed from a linear interpolation of the IH displacement data $\left(C_{\text {video }}=\right.$ linear interp. $\left(\operatorname{Disp}_{I H}\left(t_{n}, t_{n+1}\right)\right)$, with $\left.-T_{\text {video }} \in\left[t_{n}, t_{n+1}\right]\right)$.

Standard uncertainty on $C_{\text {video }}$ was assessed by $\Delta C_{\text {video }}=v_{I H} \times \Delta T_{\text {video }}$, with $v_{I H}$ being the velocity of the impactor tip $\left(v_{I H}=0.12 \mathrm{~mm} \cdot \mathrm{ms}^{-1}\right)$.

The spinal cord compression ratio $\left(C R_{\text {video }}\right)$ was determined based on the baseline morphological measurements, and calculated as $C R_{\text {video }}=C_{\text {video }} / D \emptyset_{A P}$. 


\section{$1 \quad$ Statistical analysis}

2 Reproducibility of the video-based contact detection was assessed by calculating intra-observer $3\left(C_{\text {intra }}\right)$ and inter-observer $\left(C_{\text {inter }}\right)$ coefficients of variation (defined as $C O V=S D / M$, with M 4 and SD being the mean and standard deviation respectively of the resulting cord compression 5 measurement).

6 Significance of the difference between the cord compression and compression ratio assessed by the IH 7 device versus the video methods was assessed by a sign test $(\mathrm{p}<0.05)$. The bias between the 2 methods

8 was assessed by a nonparametric Bland and Altman plot (Bland and Altman, 1999) with a limit of 9 agreements of $\pm 0.1 \mathrm{~mm}$, which is a value known to cause significant differences in injury outcomes 10 (Basso et al., 2006). 


\section{Results}

2 Average and standard deviation of spinal cord anteroposterior diameter observed at the C4 level on the nine specimens was $D \emptyset_{A P}=1.45 \pm 0.08 \mathrm{~mm}$. Repeated measurements showed an intra-observer COV of $1.8 \%$ indicating a good reproducibility. Average spinal cord right-left diameter at the $\mathrm{C} 4$ level was $2.59 \pm 0.15 \mathrm{~mm}$.

The force and cord compression obtained from the IH impactor for all experiments are detailed in Figure 2A, with the exception of one contusion injury data set in which an excessive peak force (64 kDyn) was observed (see Discussion section). This data set was excluded from all following results. The mean contusion force was $F_{I H}=31.0 \pm 1.2 \mathrm{kDyn}$, for a mean cord compression of $C_{I H}=0.31 \pm$ $0.03 \mathrm{~mm}$. The corresponding mean cord compression ratio estimated from the $\mathrm{IH}$ device was $C R_{I H}=$ $1122 \pm 2 \%$

No vertebral motion or clamp movement was detected in this study, except on the specimen with excessive peak force, for which a motion of the dorsal aspect of the $\mathrm{C} 3$ vertebra was detected when the displacement of the tip was at its deepest level (see Discussion section).

Inter-observer and intra-observer variability of the proposed video contact detection were $\operatorname{COV}_{\text {inter }}=2.48 \%$ and $\operatorname{COV}_{\text {intra }}=2.78 \%$, showing reliable and reproducible contact detection. All observers indicated the contact at the spinal cord occurred within a $1 \mathrm{~ms}$ window. For all experiments, video contact detection occurred 3.4 to 5.9 ms earlier than estimated by the IH impactor.

The mean and standard deviation of the video-based cord compression measurement was $C_{\text {video }}=$ $0.88 \pm 0.10 \mathrm{~mm}$, as compared to $C_{I H}=0.31 \pm 0.03 \mathrm{~mm}$ estimated by the IH impactor. Standard uncertainty on compression were $\Delta C_{\text {video }}=0.07 \mathrm{~mm}$ for the AOS X-VIT acquisitions, and $\Delta C_{\text {video }}=$ $0.02 \mathrm{~mm}$ for the Photron SA3 acquisitions. Figure 2B present a comparison between the video-based and IH compression measurements showing no agreement between the two methods.

Based on video analysis, the mean compression ratio and standard deviations was $C R_{\text {video }}=61 \pm 7 \%$. 
1 The discrepancies between the two contact detection methods led to significant differences in cord

2 compression and compression ratio measurements $(\mathrm{p}=0.031)$.

3 The Bland \& Altman plot of compression values with $0.1 \mathrm{~mm}$ limits of agreement revealed that the

4 bias between the two measurement methods was not due to systematic errors, but to random errors

5 (Figure 2D). Discrepancies between video-based and IH device-based results are neither constant nor

6 proportional, and are thus not predictable. 


\section{Discussion}

2 This work proposed an additional set-up to experimental contusion models to objectively improve the

3 evaluation of the spinal cord compression. Three major points were highlighted.

4 - Under our experimental spinal cord contusion set up (30 kdyn, IH $0.6 \mathrm{~mm}$ diameter tip), the

5 Infinite Horizon impactor's contact detection led to an underestimation of the spinal cord compression.

6 The force threshold of $20 \mathrm{kDyn}$ triggering the $\mathrm{IH}$ measurement of the cord compression, may have

7 provided reliable data for rat models, for which the IH impactor has mainly been designed, and for

8 which the contusion force values are between 100 and 200 kDyn (Precision Systems and

9 Instrumentation, 2012). However, this threshold value did not provide appropriate sensitivity for a

10 mouse model with a suggested force range of 30-70 kDyn (Precision Systems and Instrumentation,

11 2012). Thus, the contact detection threshold should ideally be lowered for the mouse model using

highly sensitive load cell, as needed. The method investigated in this work could be considered as an alternative, or supplement, to upgrading the IH device. Furthermore, comparison of this video-based method with the contact detection methods of the NYU-MACSIS and ESCID impactors would also be interesting and could be explored in future research.

- $\quad$ Although the video-based assessment of cord compression seemed to provide a more widely dispersed distribution than $\mathrm{IH}$ measurements (Figure 2C), the relative standard deviation of both measurement data $(R S D=S D /$ Mean $)$ reached the same result of $11 \%$. Measurement uncertainties related to the different sampling frequencies of the cameras used in this study were an order of magnitude less than the differences in measured compression values between the two methods, indicating that the use of two different cameras does not invalidate the presented results. Added to the good repeatability of the visual contact detection, results of this study showed that the proposed setup provides the ability to obtain a more accurate quantification of spinal cord compression during a contusion injury. Hence, this work could be further used to improve the analysis of compression ratios obtained within an experimental campaign. 
1 impactor (64kDyn), which was more than twice the target value. In this case, ventral motion of the C3

2 vertebra was clearly visible on the video recording while the clamps remained stationary. For this

3 specimen, the video-based compression ratio was $107 \%$. It is likely that the IH impactor completely

4 compressed the spinal cord and hit the opposite wall of the spinal canal. This unexpected impact

5 would explain both the peak force value and the vertebral motion. Impact between the impactor tip and

6 the spine may partly explain the failures of the IH clamping system previously reported (Sharif-

$7 \quad$ Alhoseini et al., 2017).

8 This study presents some limitations. First, given the camera placement, it is possible for the contusion 9 tip to contact the cord before visual detection. Thus, differences between the real spinal cord 10 compression and the compression value given by the impactor might be even greater than estimated in 11 this study. Given the impact speed of the IH device and the sampling frequencies of the cameras, a potential occurrence of contact one frame before visual detection would lead to a maximum error in compression assessment of $0.12 \mathrm{~mm}$ and $0.04 \mathrm{~mm}$ at $1000 \mathrm{fps}$ and $3000 \mathrm{fps}$ respectively. The use of a second camera could correct this limitation. Secondly, it should be noted that the highlighted relative errors of the IH cord compression assessment cannot be transposed to other data sets, especially if the impactor tip diameter differs from this study. Additional experimental investigation could be useful to compare the two methods of contact detection for a range of contusion forces and confirm the method robustness.

In conclusion, despite some limitations, this study discloses a major source of bias in a common mouse contusion model, which may consequently explains some of the reported data discrepancies in the literature. Furthermore, applying the proposed setup could improve the accuracy of spinal cord compression measurements and thus, improve the reproducibility in mouse contusion models. 


\section{Acknowledgments}

2 The authors acknowledge the assistance of Max Py from the LBA for the video acquisitions. This

3 work was supported by the French Institut pour la Recherche sur la Moelle épinière et l'Encéphale,

4 the Institut Français des sciences et technologies des transports, de l'aménagement et des réseaux,

5 and the A*MIDEX project (ANR-11-IDEX-0001-02).

\section{Declaration of interest}

7 Conflicts of interest: none. 


\section{$1 \quad$ Figures}
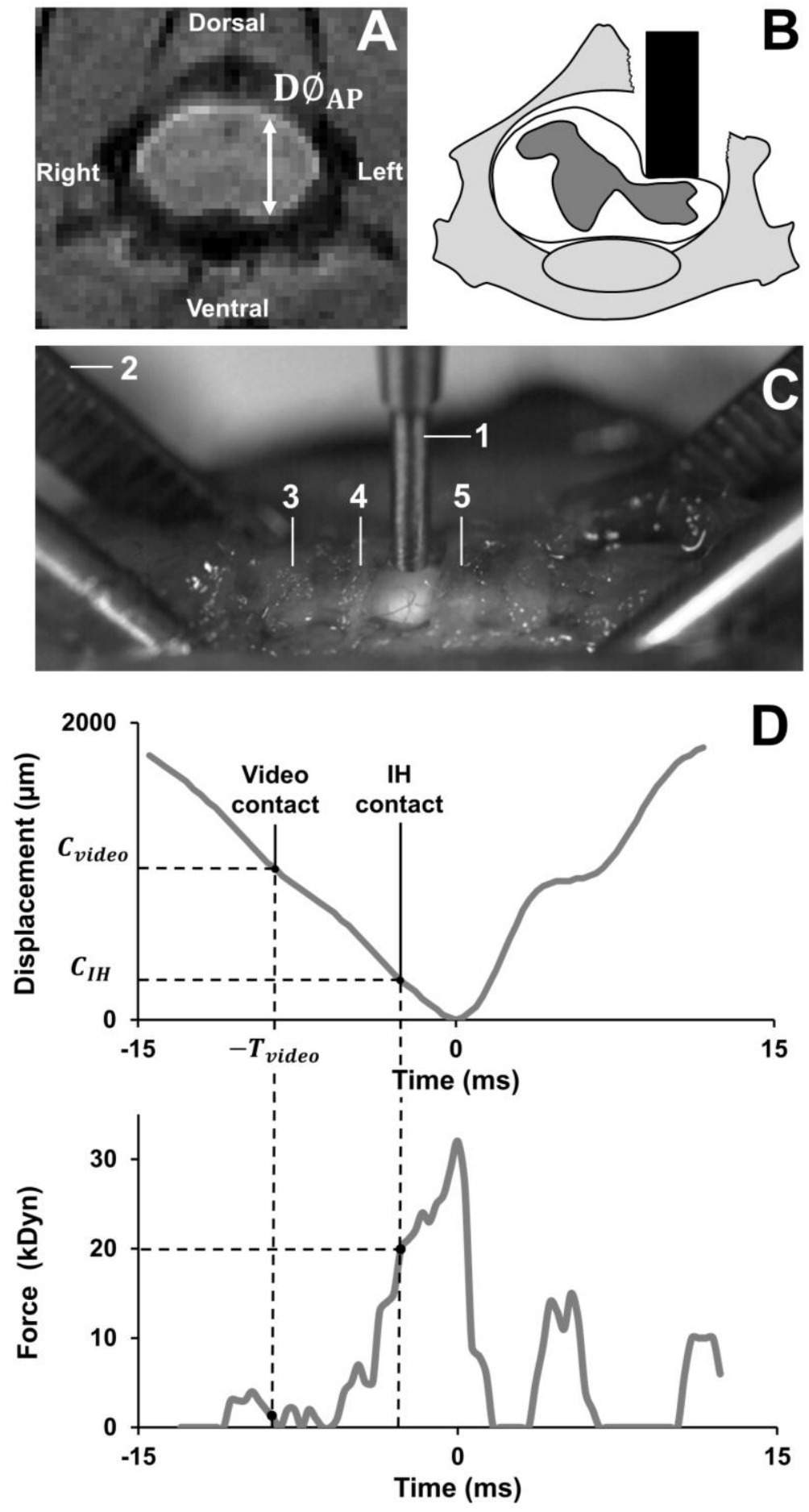

3 Figure 1 - Representative MRI acquisition (Callot et al. 2007) for antero-posterior diameter $\left(D \emptyset_{A P}\right)$

4 measurement of C4 spinal cord (A). Diagram of the contused spinal cord with representive

5 laminectomy extent (B). Representative frame of a Photron SA3 acquisition 1 - impacting tip. 2-

6 vertebral clamp. 3 - C3 lamina. 4 - partial C4 lamina. 5 - C5 lamina. (C). Representative Infinite

$7 \quad$ Horizon tip displacement acquisition $\operatorname{Disp}_{I H}(t)$ and load acquisition $F_{I H}(t)(\mathbf{D})$. 
A

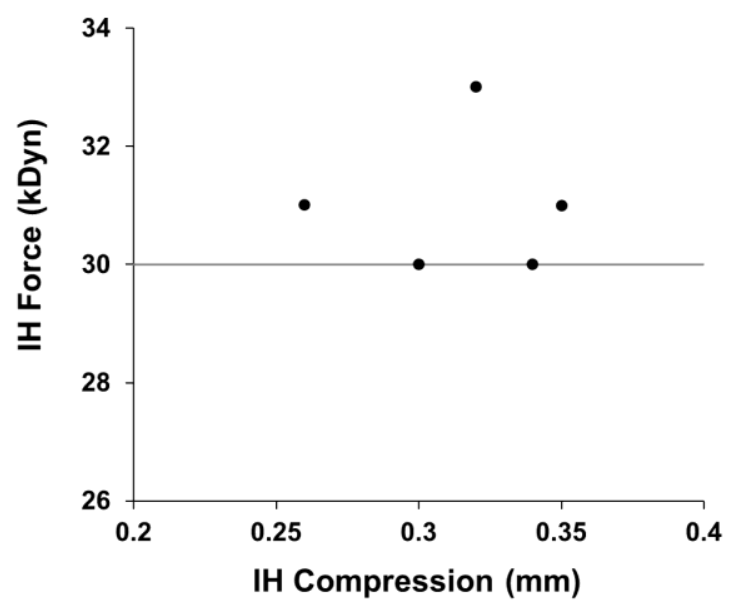

C

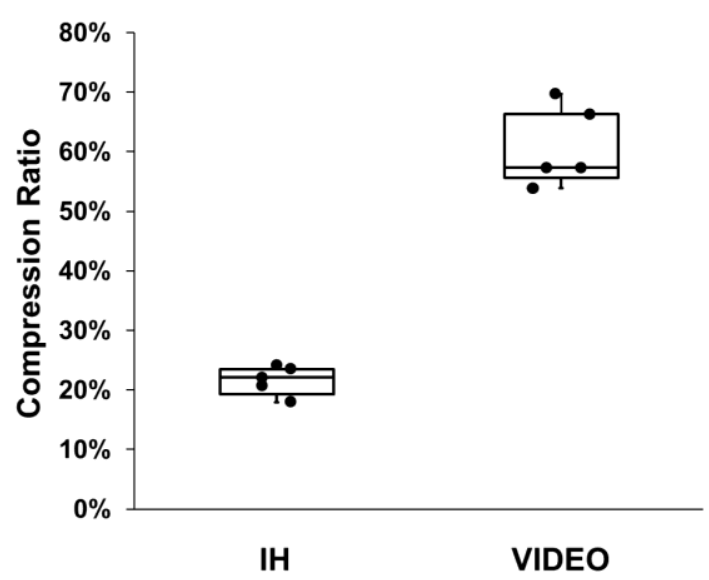

B

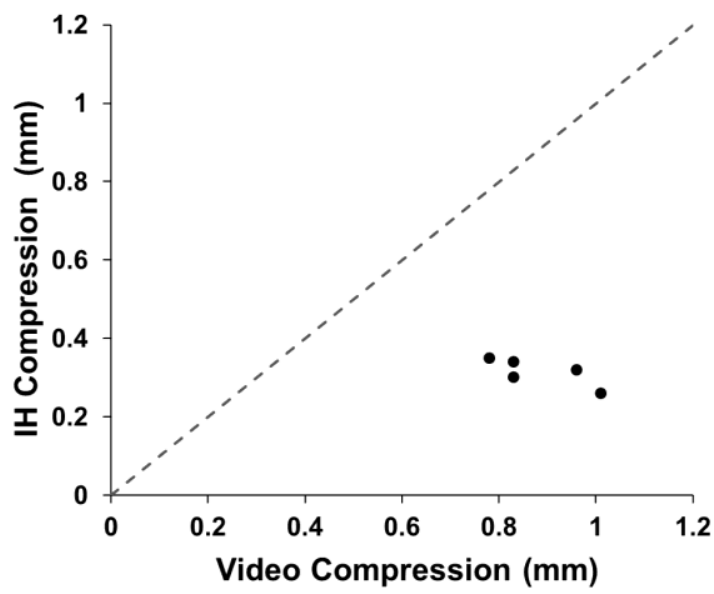

D

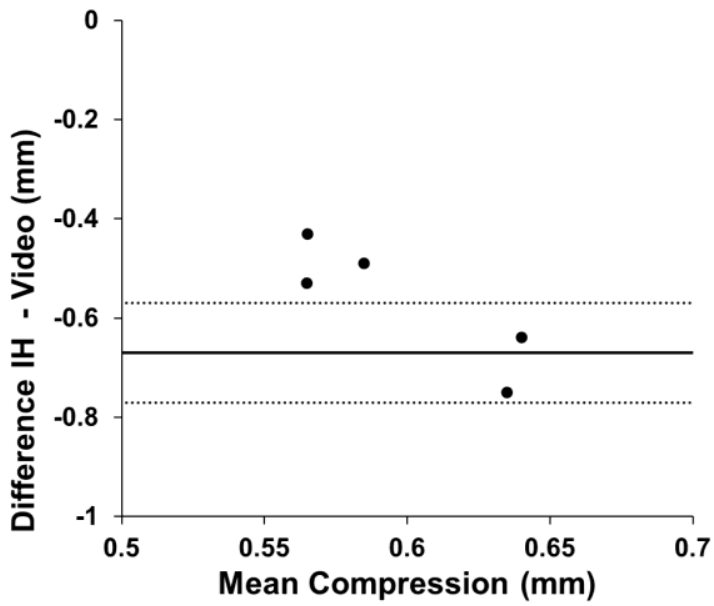

2 Figure 2 - (A) IH-based force-displacement results. (B) Plot of IH-based compression values vs. the

3 video-based compression values with the line of equality (- - - ). (C) Box plot of compression ratio

4 results according to the two methods of contact detection. (D) Bland \& Altman plot with the mean

5 difference $(-)$ and the $0.1 \mathrm{~mm}$ limits of agreement (.....) of compression results. 


\section{References}

Basso, D.M., Fisher, L.C., Anderson, A.J., Jakeman, L.B., Mc Tigue, D.M., Popovich, P.G., 2006. Basso Mouse Scale for Locomotion Detects Differences in Recovery after Spinal Cord Injury in Five Common Mouse Strains. J. Neurotrauma 23, 635-659. doi:10.1089/neu.2006.23.635

Bland, J.M., Altman, D.G., 1999. Measuring agreement in method comparison studies. Stat Methods Med Res 8, 135-160. doi:10.1177/096228029900800204

Callot, V., Duhamel, G., Cozzone, P.J., 2007. In vivo mouse spinal cord imaging using echo-planar imaging at 11.75 T. MAGMA 20, 169-173. doi:10.1007/s10334-007-0079-x

Engesser-Cesar, C., Anderson, A.J., Basso, D.M., Edgerton, V.R., Cotman, C.W., 2005. Voluntary wheel running improves recovery from a moderate spinal cord injury. J. Neurotrauma 22, 157-171. doi:10.1089/neu.2005.22.157

Gruner, J.A., 1992. A Monitored Contusion Model of Spinal Cord Injury in the Rat. J. Neurotrauma 9, 123-128. doi:10.1089/neu.1992.9.123

Jakeman, L., Guan, Z., Wei, P., Ponnappan, R., 2009. Traumatic Spinal Cord Injury Produced by Controlled Contusion in Mouse. J. Neurotrauma 17, 299-319. doi:10.1089/neu.2000.17.299

JCGM 100:2008, 2008. 4.3 - Type B evaluation of standard uncertainty, in: Evaluation of Measurement Data - Guide to the Expression of Uncertainty in Measurement.

Ma, M., Basso, M., Walters, P., Stokes, B., Jakeman, L., 2001. Behavioral and histological outcomes following graded spinal cord contusion injury in the C57B1/6 mouse. Exp. Neurol. 169, 239254. doi:10.1006/exnr.2001.7679

Myers, S.A., Andres, K.R., Hagg, T., Whittemore, S.R., 2014. CD36 deletion improves recovery from spinal cord injury. Exp. Neurol. 256, 25-38. doi:10.1016/j.expneurol.2014.03.016

Precision Systems and Instrumentation, 2012. Infinite Horizon Impactor Version 5.0.6 - User's Manual.

Scheff, S.W., Rabchevsky, A.G., Fugaccia, I., Main, J.A., Lumpp, J.E., 2003. Experimental modeling of spinal cord injury: characterization of a force-defined injury device. J. Neurotrauma 20, 179-193. doi:10.1089/08977150360547099

Schneider, C.A., Rasband, W.S., Eliceiri, K.W., 2012. NIH Image to ImageJ: 25 years of Image Analysis. Nat Methods 9, 671-675. doi:10.1038/nmeth.2089

Sekhon, L.H., Fehlings, M.G., 2001. Epidemiology, demographics, and pathophysiology of acute spinal cord injury. Spine 26, S2-12. doi: 10.1097/00007632-200112151-00002

Sharif-Alhoseini, M., Khormali, M., Rezaei, M., Safdarian, M., Hajighadery, A., Khalatbari, M.M., Safdarian, M., Meknatkhah, S., Rezvan, M., Chalangari, M., Derakhshan, P., RahimiMovaghar, V., 2017. Animal models of spinal cord injury: a systematic review. Spinal Cord 55, 714-721. doi:10.1038/sc.2016.187 
1 Steward, O., Popovich, P.G., Dietrich, W.D., Kleitman, N., 2012. Replication and reproducibility in spinal cord injury research. Exp. Neurol. 233, 597-605. doi:10.1016/j.expneurol.2011.06.017 